\title{
Role of Cell Surface Lipids and Thiol-Disulphide Exchange Pathways in Regulating the Encryption and Decryption of Tissue Factor
}

\author{
Shabbir A. Ansari ${ }^{1} \quad$ Usha R. Pendurthi $^{1} \quad$ L. Vijaya Mohan Rao ${ }^{1}$ \\ ${ }^{1}$ Department of Cellular and Molecular Biology, The University of \\ Texas Health Science Center at Tyler, Tyler, Texas, United States \\ Thromb Haemost 2019;119:860-870.

\begin{abstract}
Address for correspondence L. Vijaya Mohan Rao, PhD, Department of Cellular and Molecular Biology, The University of Texas Health Science Center at Tyler, 11937 US HWY 271, Tyler, TX 75708, United States (e-mail: Vijay.Rao@uthct.edu).
\end{abstract}

\begin{abstract}
Tissue factor (TF), a transmembrane glycoprotein, is the cellular receptor of the coagulation factors VII (FVII) and VIla (FVIla). The formation of TF-FVIla complex triggers the initiation of the blood coagulation pathway. TF plays an essential role in haemostasis, but an aberrant expression of TF activity contributes to thrombotic disorders. In health, TF pro-coagulant activity on cells is controlled tightly to allow sufficient coagulant activity to achieve haemostasis but not to cause thrombosis. It is achieved largely by selective localization of TF in the body and encryption of TF at the cell surface. A vast majority of TF on resting cells exists in an encrypted state with minimal pro-coagulant activity but becomes pro-thrombotic following cell injury or activation. At present, the mechanisms that are responsible for TF encryption and

Keywords

- tissue factor

- factor VIla

- encryption

- decryption

- sphingomyelin

- phosphatidylserine

- thiol-disulphide exchange pathways activation (decryption) are not entirely clear, but recent studies provide important mechanistic insights into these processes. To date, externalization of phosphatidylserine to the outer leaflet and thiol-disulphide exchange pathways that either turn on and off the allosteric disulphide bond in TF are shown to play a major role in regulating TF pro-coagulant activity on cell surfaces. Recent studies showed that sphingomyelin, a major phospholipid in the outer leaflet of plasma membrane, plays a critical role in the encryption of TF in resting cells. The present review provides an overview of recent literature on the above-described mechanisms of TF encryption and decryption with a particular emphasis on our recent findings.
\end{abstract}

\section{Introduction}

Tissue factor (TF), a transmembrane glycoprotein, functions as a co-factor for coagulation factor FVII and FVIIa, and the formation of TF-FVIIa complex triggers the coagulation cascade by activating both FIX and FX through their limited hydrolysis. ${ }^{1}$ In health, TF expression is limited primarily to perivascular and epithelial cells. ${ }^{2,3} \mathrm{FVII}$ and traces of FVIIa in the circulating blood comes in contact with cell surface TF upon vascular injury, which ensures haemostasis to limit blood loss but not to cause intravascular coagulation. It is not entirely clear whether low levels of TF is also present in the

received

November 30, 2018

accepted after revision

January 21, 2019

circulating blood, and if so, the activity status of the bloodborne TF. The available evidence indicates that there may be traces of TF in blood at best, ${ }^{4,5}$ but the blood-borne TF is either fully encrypted or inhibited by plasma inhibitors. ${ }^{6}$ The infectious and inflammatory disease conditions induce TF expression, either in circulating blood cells or vascular endothelial cells, by activation of TF gene transcription. ${ }^{7}$ Induced expression of TF by monocytes in response to infection is thought to be a part of the innate immune response to limit the dissemination of pathogens by trapping them inside clots. ${ }^{8}$ Expression of TF by monocytes in sepsis leads to disseminated intravascular coagulation (DIC),

(c) 2019 Georg Thieme Verlag KG Stuttgart · New York
DOI https://doi.org/

10.1055/s-0039-1681102. ISSN 0340-6245.

Jantary 21.2019 
whereas TF expression in tumour endothelium and the release of $\mathrm{TF}^{+}$microvesicles (MVs) into circulation lead to DIC and venous thromboembolism. ${ }^{9}$

Irrespective of the cellular source of TF and whether it is induced or constitutively expressed, most of the TF expressed on the surface of resting cells exists in an 'encrypted' state with very little or no pro-coagulant activity (PCA). ${ }^{10-12}$ The encrypted TF can bind to FVIIa, but the assembled TF-FVIIa complex fails to activate FIX and FX.12,13 Activation or disruption of cells markedly enhances cell surface TF activity without altering TF antigen levels at the cell surface ('decryption'). ${ }^{10,12}$ Although we and others described the above phenomenon more than 25 years ago, ${ }^{13-16}$ still it is not entirely clear how decrypted TF differs from that of cryptic TF, and mechanisms by which cryptic TF is transformed into active (pro-coagulant or pro-thrombotic) TF. Several mechanisms have been proposed for TF decryption, and out of them, externalization of phosphatidylserine (PS) to the outer leaflet of plasma membrane and oxidation of an allosteric disulphide bond of TF appears to be two most plausible mechanisms of TF decryption. ${ }^{12,17-19}$ Several recent review articles discussed these and other potential mechanisms in-depth. ${ }^{10,12,17-23}$ The focus of this review is to update our current knowledge on TF decryption mechanisms that are believed to be the predominant mechanisms and discuss a newly identified mechanism of TF encryption and decryption that is regulated by sphingomyelin (SM) and its metabolism. In the review, a reference to the earlier publications in the literature on the subject is limited to a few selective publications that are pertinent in discussing the recent data.

\section{Membrane Phospholipid-Dependent TF Encryption and Decryption}

PS-dependent TF decryption: It is well established from in vitro studies that the expression of TF PCA requires TF association with phospholipids, ${ }^{24,25}$ and the presence of anionic phospholipids, such as PS, in the phospholipid mixture markedly increases TF PCA. ${ }^{1,14,26-29}$ A substantial amount of PS ( $\sim 30 \%$ of total phospholipids) is required for the optimal TF PCA in synthetic phospholipid vesicles. ${ }^{28}$ However, incorporation of phosphatidylethanolamine (PE) or phospholipids other than those with a choline head group in TF vesicles lowers the amount of PS required for optimal proteolytic activation of FX by the TF-FVIIa complex. ${ }^{28,29}$ In contrast, the presence of SM, which contains the choline head group, was found to reduce TF PCA in relipidated TF vesicles. ${ }^{28,30}$ These data suggest that the phospholipid composition of the cell membrane may also profoundly influence TF PCA on cell surfaces.

Although it is well established that PS markedly enhances TF PCA, the molecular mechanisms by which PS augments the TF PCA are not fully understood. The binding of FVIIa and FX to PS via the Gla ( $\gamma$-carboxyglutamic acid) domain and the spatial stabilization of the FVIIa catalytic site after its binding to TF and PS are thought to contribute to the PS-mediated enhancement of TF PCA. ${ }^{31,32}$ Recently, Ohkubo et al, ${ }^{33}$ based on the molecular dynamics (MD) simulations of the TF ectodomain on the membrane surface, suggested that specific residues in TF ectodomain could directly interact with PS head groups in the membrane. The interaction between TF and PS is thought to contribute to the optimal presentation of the TF exosite region to its protein substrates, FIX and FX, through modulation of conformation-specific changes in $\mathrm{TF}^{33}$ In supporting this hypothesis, it was shown that mutation of some of the TF residues predicted to interact with PS, particularly mutation of amino acids in or near the flexible loop from Lys ${ }^{159}$ to Gly, ${ }^{164}$ decreased the PCA of TF incorporated into PS/phosphatidylcholine (PC) liposomes. ${ }^{34}$ In a recent study, investigating the effect of various synthetic PS stereoisomers on TF PCA and using MD simulations, Mallik et $\mathrm{al}^{35}$ suggested that the chiral centres of PS play a critical role in stabilizing the catalytic triad of FVIIa bound to TF by interacting with TF and FVIIa Gla domain. At present, it is unknown whether the direct interaction between phospholipids and TF ectodomain is limited to PS head groups or head groups of other phospholipids could also directly interact with TF and alter its conformation.

Phospholipids in the cell membrane are arranged asymmetrically with outer leaflet enriched with choline phospholipids, such as PC and SM, while the anionic phospholipid PS and PE reside mostly in the inner leaflet. ${ }^{36}$ Three different classes of enzymes are involved in maintaining the asymmetry of the membrane-flippase, floppase and scramblase. $^{37,38}$ Cellular responses elaborated in a variety of diseases, such as infection, hypertension, atherosclerosis and diabetes, alter the activity of these enzymes, often resulting in the externalization of negatively charged PS to the outer leaflet of cell surface membrane. ${ }^{39-41}$

Since most of the stimuli or cellular processes that disrupt membrane asymmetry and increase the levels of PS in the outer leaflet invariably increased cell-associated TF PCA by many folds, it is widely accepted that the externalization of PS following cell activation or injury is responsible for TF decryption. ${ }^{10,12}$ The observation that blockade of PS by annexin V attenuated the increased TF PCA in most of the cases gave a further credence to the above conclusion. ${ }^{12}$ At present, it is unclear whether mechanisms that were postulated are responsible for the PS-mediated increase in TF PCA in the reconstituted system would also apply to PS-dependent decryption of TF in cells. In the reconstituted system, the PSmediated increase in TF PCA comes mainly from the lowering of $K_{\mathrm{m}}$ of FX activation, ${ }^{42}$ suggesting that the substrate binding to PS in TF vesicles plays a critical role in the enhanced TF-FVIIa activation of FX. In contrast, the increased TF PCA of decrypted TF in cell systems comes primarily from the increased $V_{\max }$ and not lowering the $K_{\mathrm{m}} \cdot{ }^{43}$ In recent studies, ${ }^{44}$ we examined whether the direct TF interaction with PS in the outer leaflet plays a role in TF decryption as indicated from the studies of molecular modelling ${ }^{33}$ and reconstitution of TF mutants. ${ }^{34}$ For this, we generated a panel of plasmid and adenoviral constructs encoding TF mutants in which the putative PS interactive sites were mutated and expressed them in two different cell types lacking endogenous TF expression or having a minimal TF expression. Analysis of TF PCA in resting cells and stimulated cells showed that select amino acid residues in 
TF ectodomain that are implicated in interacting directly with PS contribute to the TF PCA at the cell surface. However, the importance of the potential interaction between the putative PS-interacting residues of TF and cell surface membrane lipids appears to be less critical than what was observed in the reconstituted system in supporting TF activity. More importantly, the PS-dependent decryption of TF in cells was not attenuated completely even when all putative PS interactive residues of TF were mutated. Overall, these data suggest that a TF region, other than the recently identified lipid-binding region, may contribute to enhanced TF PCA following PS externalization in cells. $^{44}$

The appearance of PS on cell surfaces is commonly associated with apoptosis. However, there is clear evidence for regulated externalization of PS by non-apoptotic pathways in viable cells. ${ }^{45}$ However, the mechanisms of PS externalization in response to pathophysiologic stimuli in viable cells and how it relates to TF decryption are not much known. Recently, we found that 4-hydroxy-2-nonenal (HNE), one of the most abundant reactive aldehydes generated from oxidation of $\omega 6$ fatty acids, which is believed to play a role in the pathogenesis of atherosclerosis, ${ }^{46,47}$ induced TF decryption in monocytic cells by externalizing PS via activation of the p38 mitogenactivated protein kinase (MAPK)-dependent signalling path- way. ${ }^{48}$ A follow-up study revealed that HNE induces PS externalization via two distinctive independent pathways-reactive oxygen species (ROS)-dependent and thioredoxin reductase (TrxR)/thioredoxin (Trx) system-dependent. ${ }^{49}$ TrxR/Trxdependent pathway appears to modulate PS externalization by a novel signalling pathway involving the activation of apoptosis signal-regulating kinase 1 , mitogen-activated protein kinase kinase 3/6 and p38 MAPK, whereas ROS-dependent externalization of PS was independent of p38 MAPK activation. Externalization of PS by both the pathways contributed to TF decryption in monocytic cells (-Fig. 1). ${ }^{49}$

In a recent study, Ebert et $\mathrm{al}^{50}$ showed that dysregulated redox regulation in paraoxonase-2 (Pon2) deficiency causes vascular inflammation and abnormalities in blood coagulation. Isolated endothelial cells from Pon $2^{-1-}$ mice displayed increased TF activity in vitro without altering TF protein expression levels. Annexin V binding studies showed increased PS levels in the outer leaflet of the plasma membrane of endothelial cells derived from Pon $2^{-1-}$ mice relative to wildtype mice. Administration of anti-TF antibodies or antioxidant $\mathrm{N}$-acetylcysteine normalized the pro-coagulant state of PON2 deficiency. Bone marrow transplantation studies demonstrated that endothelial cells, not haematopoietic cells, were responsible for the pro-thrombotic phenotype of Pon $2^{-1-}$ mice.

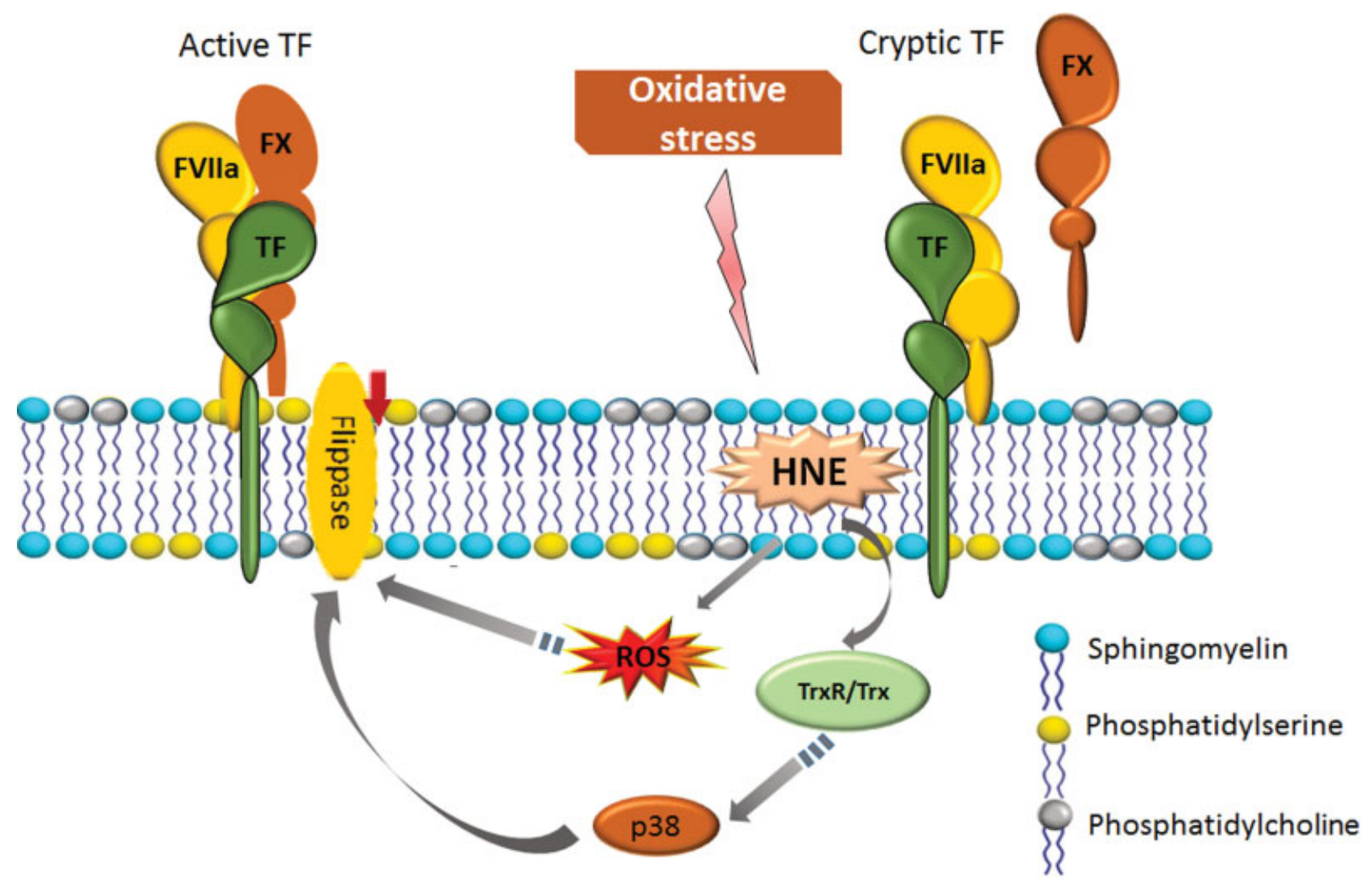

Fig. 1 Externalization of phosphatidylserine to the outer leaflet in response to a perturbing cell stimulus contributes to tissue factor (TF) decryption. Oxidative stress causes lipid peroxidation and produces reactive aldehydes, such as 4-hydroxy-2-nonenal (HNE). HNE induces reactive oxygen species (ROS) generation and inactivates the thioredoxin reductase/thioredoxin (TrxR/Trx) system. Inactivation of TrxR/Trx results in the activation of p38 mitogen-activated protein kinase (MAPK). HNE-induced p38 MAPK activation and ROS generation could lead independently to the inhibition of flippase activity. Inhibition of the flippase activity leads to the externalization of phosphatidylserine (PS). The interaction between TF-factor VIla (FVIIa) and the externalized PS transforms cryptic TF to active TF by altering the conformation of the TF-FVIIa complex to a more favourable form of interacting with protein substrate factor $\mathrm{X}$. 
Overall, these data suggest that increased oxidative stress in Pon $2^{-1-}$ mice leads to TF decryption in endothelial cells via PS externalization. Earlier studies showed that PON2 translocates to the plasma membrane in response to oxidative stress and inhibits lipid peroxidation in the plasma membrane. ${ }^{51}$ Therefore, it is possible that PON2 may be suppressing TF activity in endothelial cells by counteracting lipid peroxidation under conditions of oxidative stress. As discussed earlier, lipid peroxidation products induce PS externalization and TF decryption. ${ }^{48,49}$

Although PS externalization plays a critical role in cellular TF activation, PS exposure alone may not be sufficient to activate TF optimally. Several studies showed that the extent of TF activation is not strictly correlated with PS exposure. ${ }^{43,52-54}$ In many cases, even saturating concentrations of annexin $V$ or lactadherin that block PS failed to completely neutralize enhanced TF PCA associated with TF activation. ${ }^{43,55-57}$ These data indicate the involvement of additional molecular mechanisms in TF activation.

SM-mediated TF encryption: The reason for the existence TF in an encrypted state on cells could be due to lack of PS in the outer leaflet of the plasma membrane in resting cells, or a specific membrane phospholipid present in the outer leaflet of resting cells exerts an inhibitory effect on TF PCA. In the outer leaflet of the mammalian plasma membrane, SM contributes up to $50 \%$ of the total phospholipids present on the cell surface. ${ }^{58}$ A cursory comparison of various membrane lipids on TF activity in a reconstituted system showed a lower TF coagulant activity when TF was reconstituted in phospholipid vesicles containing SM. ${ }^{28}$ Our recent studies confirmed this finding by showing that SM suppresses TF PCA without affecting FVIIa binding to TF or the amidolytic activity of assembled TF-FVIIa complexes. ${ }^{30}$ This suggested that SM in the outer leaflet of the plasma membrane could be responsible for TF encryption in resting cells. Consistent with this possibility, hydrolysis of SM by exogenous bacterial sphingomyelinase (b-SMase), which removes the choline head group from SM, increased the cell surface TF PCA by many folds in macrophages. ${ }^{30}$ These data indicate that SM plays a critical role in maintaining an encrypted state of TF in resting cells and the breakdown of SM in the membrane releases TF from its encryption. It is important to note that SM hydrolysis-induced TF decryption was independent of PS as SM hydrolysis by bSMase did not result in PS externalization. ${ }^{30}$

SM metabolism is altered in many thrombotic disease settings, including atherosclerosis, diabetes, sepsis and cancer. ${ }^{59-63}$ It raises the possibility that SM metabolism may play a key role in TF encryption/decryption. Supporting this possibility, we found that TF decryption induced by adenosine triphosphate (ATP) activation of $\mathrm{P} 2 \times 7$ receptor $(\mathrm{P} 2 \times 7 \mathrm{R})$ signalling pathway ${ }^{54}$ was the resultant of SM hydrolysis in the outer leaflet (-Fig. 2). ${ }^{30}$ Our studies ${ }^{30}$ and others $^{64}$ showed that ATP induces the translocation of acid-SMase (ASM) from lysosomes to the outer leaflet that would breakdown SM in the outer leaflet. The observation that the knockdown of ASM with small interfering ribonucleic acid or inhibitors attenuate ATP-induced hydrolysis of SM and TF decryption provides strong confirmation that SM metabolism plays an important role in regulating TF encryption and decryption. ${ }^{30}$ At present, it is unclear how SM suppresses TF PCA, and how the hydrolysis of SM leads to increased TF activity. The tight interaction between SM and cholesterol through hydrogen bonding was thought to be the basis for raft formation. ${ }^{65}$ The membrane rafts are known to play a critical role in facilitating protein-protein and protein-lipid interactions and influence many cellular functions. ${ }^{66}$ Ultrastructural localization of TF in a variety of cell types showed that a fraction of TF in these cells is associated with lipid rafts/caveolae. ${ }^{67-70}$ It has been suggested that lipid raft/caveolae-associated TF represents the encrypted form, which can be activated rapidly by disruption of these structures. ${ }^{67,71,72}$ It is conceivable that TF decryption induced by SM hydrolysis may involve disruption of lipid rafts. It is also possible that ceramide, generated from SM hydrolysis, could bind cholesterol and form ceramide-rich platforms that alter the membrane curvature and integrity. ${ }^{73,74}$ Based on recent observations that TF ectodomain could directly interact with PS and this interaction modulates TF-FVIIa conformation to be more favourable to FX activation, ${ }^{34}$ one could envisage a direct interaction between TF and SM, which would keep TFFVIIa conformation unfavourable to its interaction with its protein substrates.

\section{Role of Thiol-Disulphide Exchange Reactions in Regulating TF Activation}

Disulphide bonds play a critical role in the folding, stabilization and maturation of extracellular domain of proteins, especially the secretory and the membrane-bound proteins. ${ }^{75,76} \mathrm{TF}$ is a transmembrane protein that contains four cysteine residues in its extracellular domain capable of forming two disulphide bonds. The N-terminal domain contains cysteines at residues 49 and 57, while the other pairs, Cys residues at 186 and 209, are at the membrane proximal region. Cys ${ }^{186}-\mathrm{Cys}^{209}$ bond has a hallmark RHStaple configuration, the characteristic of allosteric disulphide bonding. ${ }^{56,77,78}$ Allosteric disulphide bonds are dynamic and can be cleaved reductively by redox enzymes or be engaged in thiol-disulphide exchanges that regulate the function of the protein. ${ }^{78}$ Several studies in recent years indicated that thiol/disulphide switch in the Cys ${ }^{186}$-Cys $^{209}$ plays a key role in TF encryption and decryption ${ }^{18-20}$ (- Fig. 3A).

Chen et al were the first to show that TF expressed on cell surfaces contained free thiols and cellular activation with ionomycin or $\mathrm{HgCl}_{2}$ depleted free thiols in $\mathrm{TF}^{56}$ Thiol alkylators, such as N-ethylmaleimide that can block unpaired cysteine residues to form disulphide bond, inhibited the ability of ionomycin and $\mathrm{HgCl}_{2}$ to increase TF activity. ${ }^{56}$ Ionomycin and $\mathrm{HgCl}_{2}$ treatments also resulted in a greater proportion of HL60 cells binding to V1C7 TF monoclonal antibody $(\mathrm{mAb})$ that recognizes an epitope within residues 181-214 of $\mathrm{TF}^{56}$ These data led to a hypothesis that the Cys $^{186}$ and Cys $^{209}$ residues exist in reduced thiol form in cryptic TF and decryption involves the formation of the disulphide. It had been suggested that the formation of $\mathrm{Cys}^{186}-\mathrm{Cys}^{209}$ disulphide bond changes the conformation of TF that facilitates the binding of substrates FIX and FX. ${ }^{56}$ 


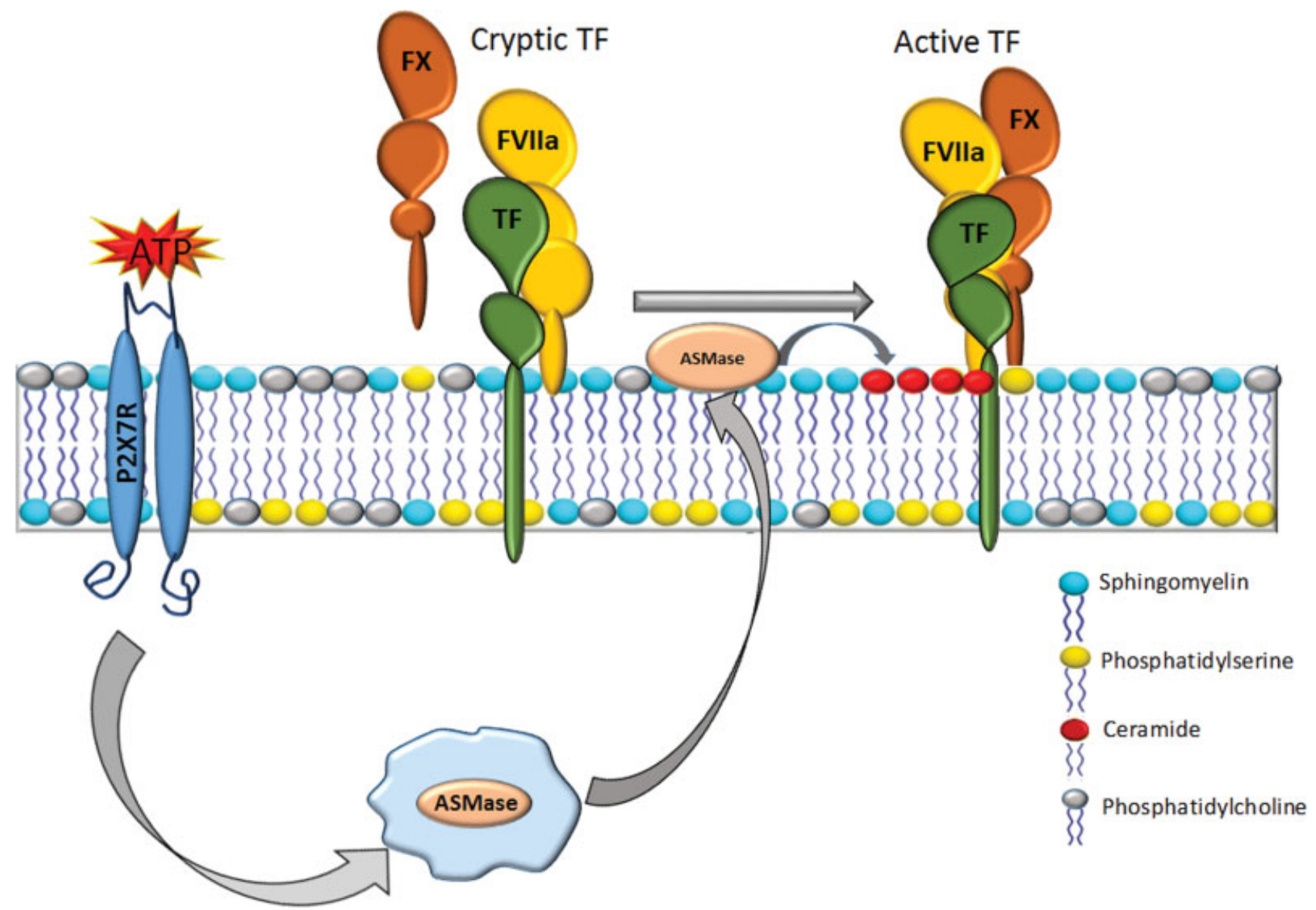

Fig. 2 Sphingomyelinase-mediated tissue factor (TF) decryption. The presence of a high molar concentration of sphingomyelin (SM) in the outer leaflet of the plasma membrane inhibits TF pro-coagulant activity on the cell surface, thus maintaining TF in an encrypted state in resting cells. Cell activation, such as adenosine triphosphate (ATP)-induced signalling pathway via $\mathrm{P} 2 \times 7$ receptor $(\mathrm{P} 2 \times 7 \mathrm{R})$ or the inflammatory mediators-induced signalling pathways would lead to the translocation of acid-sphingomyelinase (ASM) from intracellular compartments to the outer leaflet. ASM hydrolyzes SM in the outer leaflet, which removes the SM's inhibitory effect on TF and thus resulting in increased TF pro-coagulant activity.

Mutagenesis of $\mathrm{Cys}^{186}$ and $\mathrm{Cys}^{209}$ residues, which prevents the formation of a $\mathrm{Cys}^{186}{ }_{-\mathrm{Cys}}{ }^{209}$ disulphide bond, severely impaired TF PCA in several cell types, and this was taken as compelling evidence in supporting the above hypothesis. ${ }^{79-82}$ However, low expression of the mutants at the cell surface and their ability to be activated by ionomycin, to a similar fold to that of wild-type TF, suggested that severe impairment in TF PCA associated with $\mathrm{Cys}^{186}$ and $\mathrm{Cys}^{209}$ mutation may reflect the impaired TF synthesis, folding or membrane trafficking rather than just failure to form allosteric disulphide bond..$^{83}$ However, baby hamster kidney cell transfectants expressing similar levels of TF-Cys ${ }^{209}$ mutant as of wild-type TF showed a complete loss of TF coagulant function, ruling out the above possibility. ${ }^{82}$ Similar data were also obtained with stable transfectants of murine TF Cys ${ }^{190}$ and Cys ${ }^{213}$ mutants (corresponding to $\mathrm{Cys}^{186}$ and $\mathrm{Cys}^{209}$ residues, respectively, in human TF), which expressed equal levels of surface TF as of wild-type TF. Both single and double Cys to Ala replacements showed a severe reduction in TF PCA. ${ }^{84}$

Recently, MD simulations were used to understand how the $\mathrm{Cys}^{186}{ }^{18 y s^{209}}$ disulphide bonding could influence TF-FVIIa coagulant activity. ${ }^{85,86}$ Simulations of wild-type and TF mutants showed no significant global structural changes in
TF upon ablation of the allosteric disulphide bond, but there were local changes right in the vicinity of the respective mutation. ${ }^{85}$ Based on the MD simulations, it was suggested that FVII binding to TF involved direct detection of the disulphide instead of disulphide-dependent structural changes elsewhere in $\mathrm{TF}^{85}$ In a recent study, Prasad et al performed MD simulations of both oxidized and reduced $\left(\mathrm{Cys}^{186}-\mathrm{Cys}^{209}\right)$ forms of membrane-bound TF and TF-FVIIa complex to address how the allosteric disulphide modulate TF structure. ${ }^{86}$ Their simulation data showed that the $\mathrm{Cys}^{186}-\mathrm{Cys}^{209}$ bond is responsible for maintaining the integrity and conformation of both free TF and TF-FVIIa. The data also showed that the allosteric disulphide bond enhances FVIIa binding to TF and modulates the structure of FVIIa in the complex. In addition, the presence of the disulphide bond allows more number of TF residues to interact with lipid. ${ }^{86}$ Biochemical studies using purified placenta TF showed that reduction of the allosteric disulphide bond in TF did not affect TF binding to FVIIa but resulted in a complete loss of TF co-factor function. ${ }^{87}$ Although there is a discrepancy between MD simulation and functional data on the role of allosteric disulphide bond on FVIIa recognition, both the datasets support the hypothesis that $\mathrm{Cys}^{186}-\mathrm{Cys}^{209}$ bond plays a critical role in regulating TF coagulant activity. 

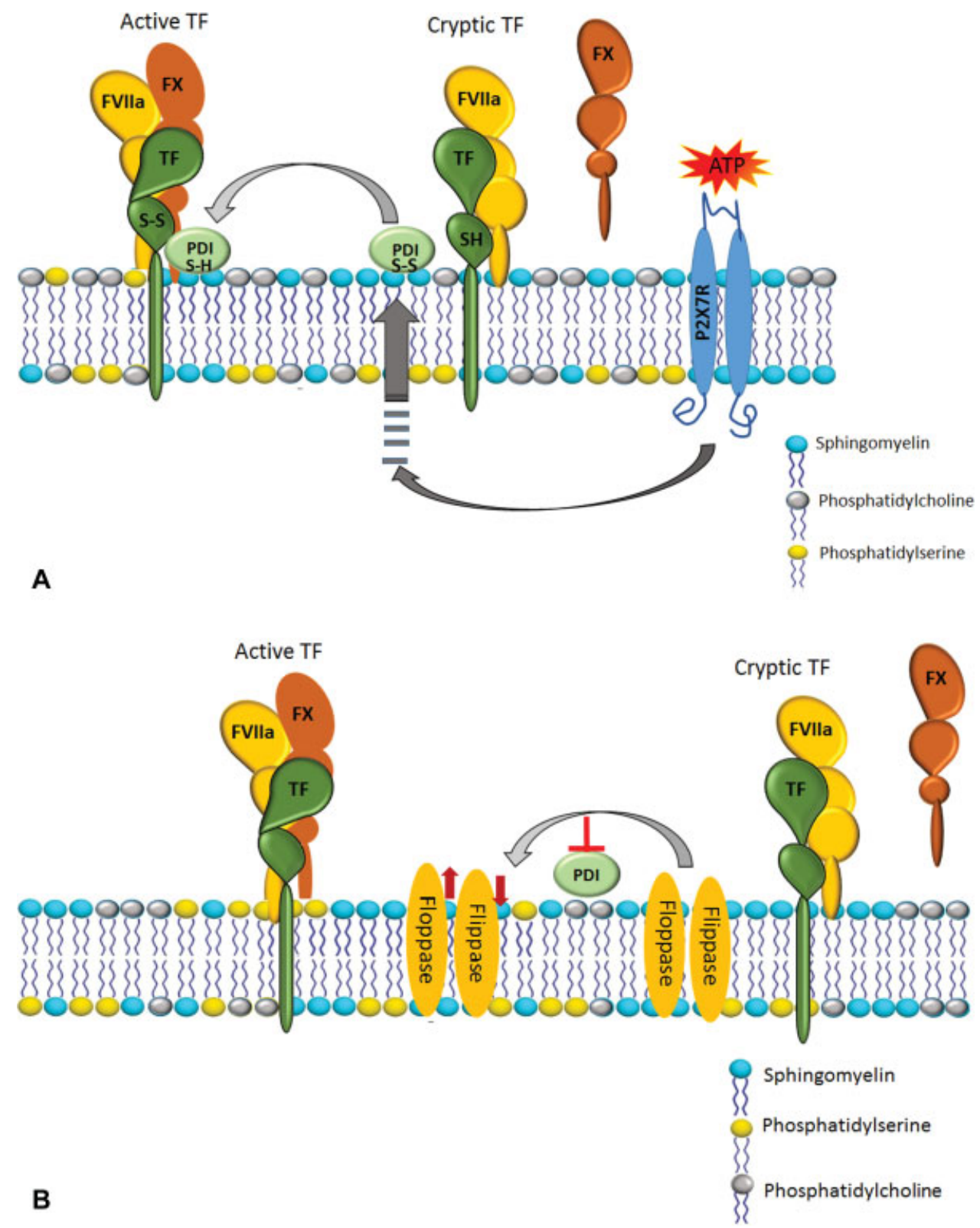

Fig. 3 Mechanisms of protein disulphide isomerase (PDI)-mediated tissue factor (TF) decryption. (A) Cryptic TF contains free thiols. Cell activation by adenosine triphosphate (ATP) or other mediators results in the secretion of PDI and/or facilitates cell surface-associated PDIdependent thiol-disulphide exchange reactions that lead to the formation of Cys ${ }^{186}$-Cys209 disulphide bond. The formation of Cys ${ }^{186}$-Cys ${ }^{209}$ disulphide bond results in a conformational change in TF-factor VIla (FVIla), favouring its interaction with factor X. (B) PDI, through its oxidoreductase activity, also modulates phosphatidylserine (PS) dynamics at the cell surface. Inhibition of PDI at the cell surface in endothelial cells was shown to inhibit the flippase and activate the floppase that results in the externalization of PS. PDI-dependent thiol-disulphide exchange reactions could also externalize PS. PDI-dependent thiol-disulphide exchange reactions that can switch on/off disulphide bonding in TF and modulate PS dynamics may closely cooperate in regulating TF pro-coagulant activity.

\section{Role of Protein Disulphide Isomerase in Regulating TF Activation}

Protein disulphide isomerase (PDI) is an oxidoreductase enzyme found abundantly in the endoplasmic reticulum of eukaryotic cells where it catalyzes disulphide bond formation between cysteine residues of proteins. ${ }^{88}$ The presence of PDI on the surface of cells $s^{49,53,79}$ or secretion from endothelial cells or platelets upon activation have been reported..$^{80,89}$ Ahamed et al showed that PDI associated with the extracellular domain of TF on the surface of HaCat keratinocytes was responsible for maintaining TF in a low pro-coagulant state. ${ }^{79}$ Treatment of the cells with $\mathrm{HgCl}_{2}$ dissociated PDI from TF and induced TF activation.

In vivo studies of Reinhardt et $\mathrm{al}^{80}$ showed that PDI was released at the injury site from adherent platelets and disrupted vessel wall cells and the inhibition of PDI decreased TFtriggered fibrin generation in various in vivo murine models of thrombus formation. This study also showed that cysteine 209 residue of TF constitutively existed in a glutathionylated form which maintains TF in cryptic form and that PDI converts cysteines from the glutathionylated to disulphide state. ${ }^{80}$ 
Furthermore, both soluble and membrane-anchored TF were shown to contain free thiols, and the exposure of soluble TF to recombinant PDI reduced the number of free thiols suggesting that PDI induces the disulphide bond formation. In parallel studies, Cho et $\mathrm{al}^{90}$ showed a time-dependent increase of PDI in murine thrombi following laser injury. Infusion of PDI inhibitor bacitracin or a function-blocking $\mathrm{mAb}$ against PDI inhibited thrombus formation and fibrin generation. In subsequent studies, this group showed that endothelium-derived and not platelet-derived PDI contributes to thrombus formation in vivo. ${ }^{89}$

In addition to regulating TF PCA via disulphide regulatory switch, PDI can also regulate TF PCA by modulating PS dynamics (-Fig. 3B). Inhibition of PDI by neutralizing antibodies or gene silencing was shown to enhance TF activity in EA.hy926 endothelial cells. ${ }^{91}$ This study showed that inhibition of PDI decreased the flippase activity and enhanced the floppase activity. ${ }^{91}$ Our recent studies provide further support for the role of thiol exchange pathways in the regulation of PS externalization. These studies showed that phenylarsine oxide, which blocks free thiols, significantly attenuated the HNE-induced TF activation. ${ }^{49}$ However, inhibition of PDI with specific inhibitors did not affect the HNE-induced TF activation. It is possible that in addition to PDI, other thiol-disulphide exchange reactions may also play a role in TF decryption by regulating the externalization of PS at the cell surface.

It is possible that both PDI-dependent and PDI-independent thiol-disulphide exchange reactions could contribute to TF activation. Stimulation of the purinergic receptor $\mathrm{P} 2 \times 7 \mathrm{R}$ by its ligand ATP was shown to induce TF activation and generation of $\mathrm{TF}^{+} \mathrm{MV}$ from macrophages and smooth muscle cells. ${ }^{54}$ Blockade of the free thiols by 5,5-dithio-bis-(2-nitrobenzoic acid) (DTNB), a thiol alkylator, was found to inhibit the ATP-induced cellular TF decryption. Both DTNB and PDI antibody (RL-90) inhibited TF decryption, the release of $\mathrm{TF}^{+}$ MV and pro-thrombotic activity of the MV. However, DTNB, and not RL90, was found to inhibit the PS externalization upon ATP stimulation. These contrasting observations led to a conclusion that PS exposure alone was not sufficient for TF activation, the release of MV and they require PDI-dependent thiol-disulphide exchange reactions. ${ }^{54}$

The above conclusion is supported in further studies where Langer et al showed that anti-thymocyte globulin (ATG)induced complement activation results in rapid activation of TF on monocytic cells more potently than any other reported stimuli that decrypt $\mathrm{TF}^{53}$ ATG-mediated TF decryption was shown to be dependent on lipid raft integrity and complement activation and did not require maximal PS exposure. ATGmediated TF activation depends on intact surface PDI activity and requires oxidation of PDI downstream of C5 cleavage. Blockade of the free thiol groups by chemical reagents, PDI inhibitory antibody or small molecule inhibitors of PDI was found to inhibit the ATG-induced TFactivation implicating that the decryption process was dependent on PDI. Complement activation also leads to PS externalization following the C5b-7 membrane insertion. In this context, it had been suggested that PDI-dependent thiol-disulphide exchanging closely cooperates with extracellular PS to lead maximal activation of TF. ${ }^{18}$
In a more recent study, Müller-Calleja et $\mathrm{al}^{92}$ found that cofactor-independent anti-phospholipid antibodies (aPLs) also rapidly activated TF on myelomonocytic cells in a PDI-dependent fashion, but TF activation by aPLs required $\mathrm{C} 3$ activation and not C5. As observed with ATG, aPLs-induced TF activation appears to be the result of coordinated effects of surfacelocated PDI-mediated thiol-disulphide exchange reactions and PS externalization.

A recent study from Rothmeier et al provided new insights into the regulation of TF PCA at the cell surface and cellular mechanisms of $\mathrm{TF}^{+} \mathrm{MV}$ generation. This study showed that $\mathrm{TF}$ cell surface activity on primary macrophages was effectively controlled by dynamin-mediated receptor internalization. ${ }^{93}$ Inhibition of dynamin was shown to not only prevent TF internalization but also to induce the release of $\mathrm{TF}^{+} \mathrm{MV}$. Arf6 recycling pathway, which controls integrin $\alpha 4 \beta 1$ function in macrophages, was shown to regulate the surface availability of TF. Uncoupling of the TF integrin $\alpha 4 \beta 1$ complex from arf6 control following ATP stimulation of $\mathrm{P} 2 \times 7 \mathrm{R}$ was thought to be responsible for the incorporation of TF into MV. Proteome analysis of MV suggested that only MV generated from ATP stimulation, bearing PDI, showed the maturation of TF that enabled its binding to FVIla with nanomolar affinity. Although inhibition of dynamin-dependent internalization also induces MV containing pro-coagulant PS, they lack PDI and fail to produce fibrin strands in the flowing blood as it was found with ATP-generated $\mathrm{PDI}^{+} \mathrm{TF}^{+} \mathrm{MV}$. These studies suggest that exposure of pro-coagulant phospholipid is not sufficient to generate pro-thrombotic MV, but also requires PDI-dependent TF affinity maturation.

\section{Role of Thioredoxin System in Regulating TF Activation}

In addition to PDI, TrxR/Trx system is also shown to regulate TF activity in a thiol-dependent manner. Wang et al showed a reversible association between the $\mathrm{Cys}^{73}$ residue of $\operatorname{Trx}$ and $\mathrm{Cys}^{209}$ residue of TF via a disulphide bond in human serum and plasma samples. ${ }^{94}$ The binding of Trx to TF was suggested to inhibit the interaction of FVIla with TF and thereby keeping TF in a low pro-coagulant state. Knockdown of Trx in MDA-MB231 cells was shown to significantly increase TFPCA. $\mathrm{H}_{2} \mathrm{O}_{2}$-induced increased cell surface TF activity was suppressed efficiently by elevating cellular TrxR activity via selenium supplementation, whereas annexin $\mathrm{V}$ treatment minimally attenuated $\mathrm{H}_{2} \mathrm{O}_{2}$ induced TF activation. These data indicate that TrxR/Trx acts as a negative regulator of TF activity.

A recent study showed that activation of $P 2 \times 7 R$ by ATP uncouples the TrxR/Trx system and activates the inflammasome, which leads to the cellular release of reduced Trx and generation of thiol pathway-dependent pro-coagulant $\mathrm{TF}^{+}$ MV. ${ }^{95}$ The reductase function of TrxR was shown to be critically important in regulating the functions of most of the downstream signalling molecules involved in $\mathrm{TF}^{+} \mathrm{MV}$ release upon $\mathrm{P} 2 \times 7 \mathrm{R}$ activation. In a broad agreement with the above studies, we recently found that TrxR plays an important role in regulating TFactivation. Upon stimulation with HNE, TrxR activity was completely inhibited, resulting in the inhibition of Trx activity. 
Inhibition of Trx by pharmacological inhibitors resulted in p38 MAPK-dependent externalization of PS leading toTFactivation, the same pathway that is involved in the HNE-induced TF activation. These data indicate that under oxidative stress, inhibition of the Trx/TrxR system could also lead to PS-dependent TF activation.

Overall, the existing data support the concept that the TrxR/Trx system plays a critical role in regulating TF activity, but the mechanisms by which it controls TF activity are not entirely clear. The reductive function of TrxR was suggested to be essential for the secretion of $\operatorname{Trx}$ to the extracellular milieu and to mediate the changes in the thiol groups of the proteome. ${ }^{95}$ It is also possible that the TrxR/Trx system could regulate TF PCA via PDI as it was shown to be a substrate for TrxR. ${ }^{96}$ Furthermore, TrxR, by activating disulphide reductase activity of PDI, was shown to reduce disulphide bonds. ${ }^{97}$

\section{Conclusion}

Studies conducted in the past few years have identified novel mechanisms of regulation of TF PCA on cell surfaces and provided additional mechanistic details to existing mechanisms. Although TF encryption/decryption appears to be a common mechanism by which TF expressing cells control their pro-coagulant phenotype, the mechanisms of TF encryption and decryption are most likely cell type- and context- dependent. Externalization of PS in response to stimuli induces TF activation in all cell types. ${ }^{12}$ However, externalization of PS is not an absolute requirement for TF activation. ${ }^{30}$ Where PS externalization contributes toTFactivation, it alone may not be sufficient for full cellular activation of TF. ${ }^{18}$ Other pathways, such as PDI-mediated thiol-disulphide exchanges and altered SM metabolism, also play a critical role in cellular activation of TF and generation of pro-thrombotic $\mathrm{TF}^{+}$MV. Emerging evidence indicates that PDI-dependent thiol-disulphide exchange closely cooperates with extracellular PS in activating TF in monocytes. ${ }^{18,53,92}$ Our recent observation ${ }^{30}$ that showed ATP stimulation of the purinergic $\mathrm{P} 2 \times 7 \mathrm{R}$ induces the translocation of ASM to the outer leaflet and the ASM-mediated hydrolysis of SM releases TF from its inhibition from SM, coupled with earlier observations that ATP-induced activation of TF involves synergies of PDI and PS, ${ }^{18}$ suggest that the coordinated effects of SM hydrolysis, PS externalization and PDI-mediated thioldisulphide exchange pathways are responsible for full cellular activation of TF ( - Fig. 4). However, molecular links among various pathways of TF decryption are not fully known yet.

MD simulations and mutagenesis studies indicated that a direct interaction between PS head groups and TF ectodomain alters the conformation of TF to allow optimal presentation of TF exosite regions to its protein substrates. ${ }^{34}$ Although a few select TF residues that are implicated in interacting with PS were found to contribute to basal TF PCA of unperturbed cells,

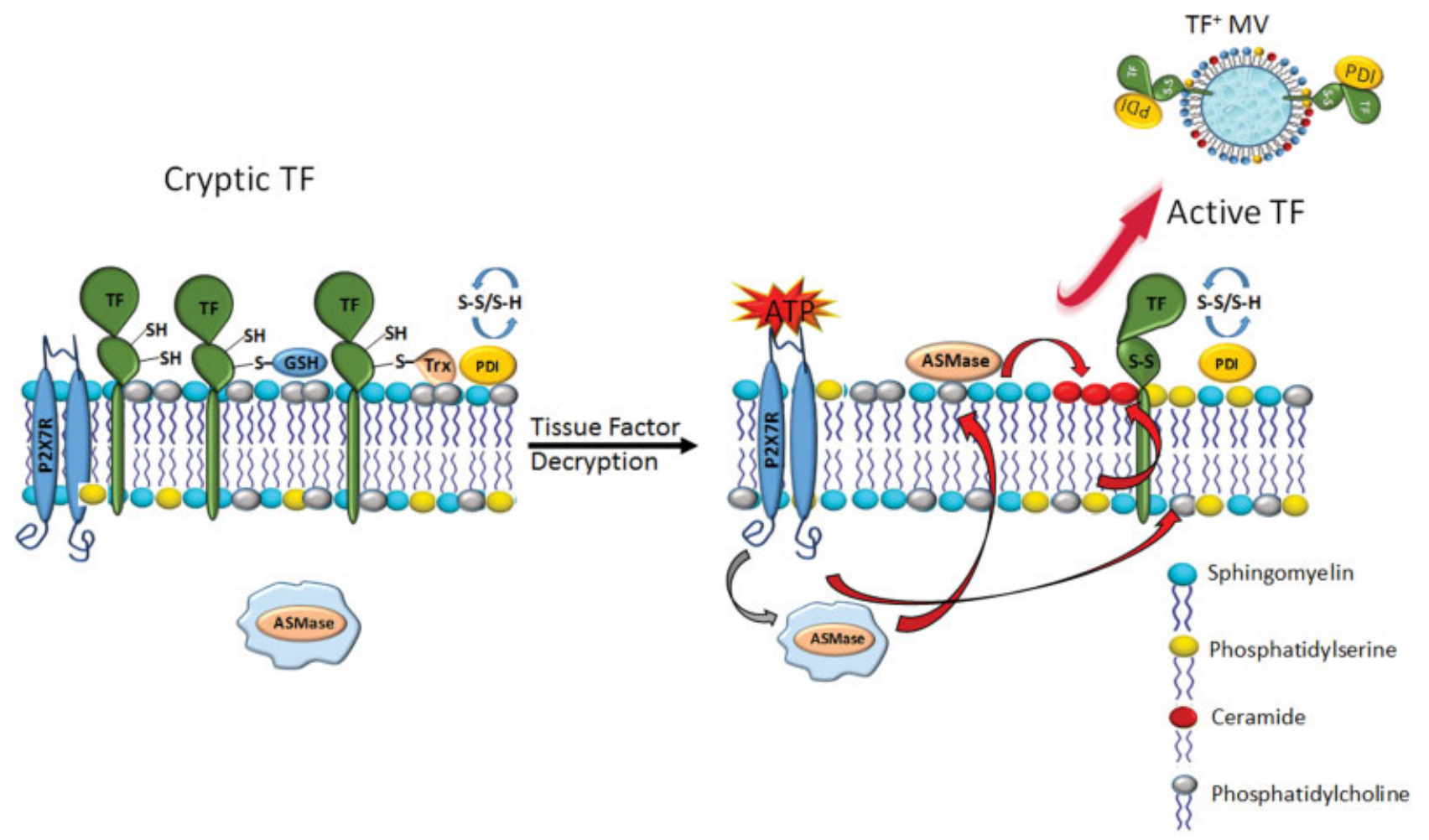

Fig. 4 A coordinated mechanism of tissue factor (TF) activation. In resting cells, TF is maintained in an encrypted state by the presence of abundant sphingomyelin (SM) in the outer leaflet, sequestration of phosphatidylserine (PS) to the inner leaflet and TF with a reduced Cys ${ }^{186}$-Cys ${ }^{209}$ allosteric disulphide bond or a mixed disulphide bond. Adenosine triphosphate (ATP) activation of the $\mathrm{P} 2 \times 7$ receptor (P2 $\times 7 \mathrm{R})$ or other cell stimulus induces cell signalling that mobilizes acid-sphingomyelinase (ASM) from lysosomal compartment to the outer leaflet of the plasma membrane. Breakdown of SM by ASM releases TF from the SM-mediated encryption and alters membrane structure and fluidity leading to PS externalization and plasma membrane blebbing and shedding. ATP activation of the P $2 \times 7 R$ also results in PS externalization and protein disulphide isomerase (PDI)-dependent thiol-disulphide exchange at the cell surface that results in the formation of Cys ${ }^{186}$-Cys ${ }^{209}$ disulphide bond in TF and alters TF conformation more favourable to the substrate binding. All events may work coordinately to fully activate TF at the cell surface and the release of $\mathrm{TF}^{+} \mathrm{MV}$. 
PS-dependent decryption of TF was largely independent of this putative interaction. ${ }^{44}$ Therefore, the question of how PS contributes to TF decryption remains to be answered.

Most of our studies on the role of phospholipids in TF encryption and decryption were limited to the role of PS. We need to expand the studies to investigate the possible role of other membrane phospholipids in the regulation of TF PCA. Our recent studies that demonstrate SM in the outer leaflet of the membrane plays a critical role in maintaining TF in an encrypted state, and the hydrolysis of SM leads to TF decryp$\operatorname{tion}^{30}$ open new avenues of research in this direction. It will be interesting to investigate whether SM head groups interact directly with TF ectodomain to keep TF in cryptic conformation. The molecular simulation may become a valuable tool in exploring the roles of membrane lipids in TF encryption and decryption.

A recent analysis of the role of thiol groups in regulating TF activity yielded new insights into the role of PDI and TrxR/ Trx systems in controlling TF activity. PDI was not only found to regulate TF encryption and decryption at the cell surface but also showed to promote the affinity of TF towards FVIIa on MV. ${ }^{93}$ PDI and thiol pathways appear to control both critical determinants of TF activation, that is, Cys ${ }^{186}-\mathrm{Cys}^{209}$ disulphide bonding ${ }^{79,80}$ and externalization of PS to the outer leaflet. $^{49,91}$ The Trx system, owing to its oxidoreductase activities and cell signalling properties, has also emerged as yet another important candidate that regulates TF PCA. It has been implicated in regulating extracellular thiol proteome that is necessary for TF activation and $\mathrm{TF}^{+} \mathrm{MV}$ release. The $\operatorname{TrxR} / \operatorname{Trx}$ system is also found to regulate TF activity by regulating the externalization of $\mathrm{PS}^{49}$ or by forming mixed disulphide bonding with $\mathrm{TF}^{94}$

Finally, many components and pathways that influence TF PCA in cells have been identified. The challenge for future research is to identify molecular links among these pathways and determine their role in regulating TF activity in vivo under various pathophysiological settings. Such information would be useful not only in understanding the pathogenesis of disease but also helpful in developing targeted therapeutic approaches in controlling bleeding disorders and thrombotic events by switching on and off TF PCA at will.

\section{Authors' Contributions}

All authors contributed to the preparation and review of the article.

\section{Funding}

This work was supported by the National Heart, Lung, and Blood Institute grants HL124055 and HL107483 to L.V.M.R. and American Heart Association's post-doctoral fellowship award to S.A.A.

\section{Conflict of Interest}

None declared.

\section{Acknowledgement}

The authors are thankful to Stephanie Melton for proofreading the manuscript.

\section{References}

1 Rapaport SI, Rao LVM. The tissue factor pathway: how it has become a "prima ballerina". Thromb Haemost 1995;74(01): 7-17

2 Drake TA, Morrissey JH, Edgington TS. Selective cellular expression of tissue factor in human tissues. Implications for disorders of hemostasis and thrombosis. Am J Pathol 1989;134(05):1087-1097

3 Fleck RA, Rao LVM, Rapaport SI, Varki N. Localization of human tissue factor antigen by immunostaining with monospecific, polyclonal anti-human tissue factor antibody. Thromb Res 1990;59(02):421-437

4 Giesen PL, Rauch U, Bohrmann B, et al. Blood-borne tissue factor: another view of thrombosis. Proc Natl Acad Sci U S A 1999;96(05): 2311-2315

5 Butenas S, Bouchard BA, Brummel-Ziedins KE, Parhami-Seren B, Mann KG. Tissue factor activity in whole blood. Blood 2005;105 (07):2764-2770

6 Osterud B, Olsen JO, Bjørklid E. What is blood borne tissue factor? Thromb Res 2009;124(05):640-641

7 Osterud B, Bjorklid E. Tissue factor in blood cells and endothelial cells. Front Biosci (Elite Ed) 2012;4:289-299

8 van der Poll T, Herwald $H$. The coagulation system and its function in early immune defense. Thromb Haemost 2014;112(04):640-648

9 Grover SP, Mackman N. Tissue factor: an essential mediator of hemostasis and trigger of thrombosis. Arterioscler Thromb Vasc Biol 2018;38(04):709-725

10 Bach RR. Tissue factor encryption. Arterioscler Thromb Vasc Biol 2006;26(03):456-461

11 Kothari H, Pendurthi UR, Rao LV. Analysis of tissue factor expression in various cell model systems: cryptic vs. active. J Thromb Haemost 2013;11(07):1353-1363

12 Rao LV, Pendurthi UR. Regulation of tissue factor coagulant activity on cell surfaces. J Thromb Haemost 2012;10(11): 2242-2253

13 Le DT, Rapaport SI, Rao LVM. Relations between factor VIIa binding and expression of factor VIIa/tissue factor catalytic activity on cell surfaces. J Biol Chem 1992;267(22):15447-15454

14 Bach R, Rifkin DB. Expression of tissue factor procoagulant activity: regulation by cytosolic calcium. Proc Natl Acad Sci U S A 1990;87(18):6995-6999

15 Ploplis VA, Edgington TS, Fair DS. Initiation of the extrinsic pathway of coagulation. Association of factor VIIa with a cell line expressing tissue factor. J Biol Chem 1987;262(20):9503-9508

16 Maynard JR, Heckman CA, Pitlick FA, Nemerson Y. Association of tissue factor activity with the surface of cultured cells. J Clin Invest 1975;55(04):814-824

17 Rao LVM, Kothari H, Pendurthi UR. Tissue factor: mechanisms of decryption. Front Biosci (Elite Ed) 2012;4:1513-1527

18 Langer F, Ruf W. Synergies of phosphatidylserine and protein disulfide isomerase in tissue factor activation. Thromb Haemost 2014;111(04):590-597

19 Ruf W. Role of thiol pathways in TF procoagulant regulation. Thromb Res 2012;129(Suppl 2):S11-S12

20 Chen VM, Hogg PJ. Encryption and decryption of tissue factor. J Thromb Haemost 2013;11(Suppl 1):277-284

21 Versteeg HH, Ruf W. Thiol pathways in the regulation of tissue factor prothrombotic activity. Curr Opin Hematol 2011;18(05): 343-348

22 Zelaya H, Rothmeier AS, Ruf W. Tissue factor at the crossroad of coagulation and cell signaling. J Thromb Haemost 2018;16(10): 1941-1952

23 Kiouptsi K, Reinhardt C. Protein disulfide-isomerase - a trigger of tissue factor-dependent thrombosis. Clin Hemorheol Microcirc 2016;64(03):279-286

24 Chargaff E. Remarks on the role of lipids in blood coagulation. Arch Sci Physiol (Paris) 1948;2:269-271

25 Nemerson Y. The phospholipid requirement of tissue factor in blood coagulation. J Clin Invest 1968;47(01):72-80 
26 Bjorklid E, Storm E. Purification and some properties of the protein component of tissue thromboplastin from human brain. Biochem J 1977;165(01):89-96

27 Krishnaswamy S, Field KA, Edgington TS, Morrissey JH, Mann KG. Role of the membrane surface in the activation of human coagulation factor X. J Biol Chem 1992;267(36):26110-26120

28 Neuenschwander PF, Bianco-Fisher E, Rezaie AR, Morrissey JH. Phosphatidylethanolamine augments factor VIla-tissue factor activity: enhancement of sensitivity to phosphatidylserine. Biochemistry 1995;34(43):13988-13993

29 Shaw AW, Pureza VS, Sligar SG, Morrissey JH. The local phospholipid environment modulates the activation of blood clotting. J Biol Chem 2007;282(09):6556-6563

30 Wang J, Pendurthi UR, Rao LVM. Sphingomyelin encrypts tissue factor: ATP-induced activation of A-SMase leads to tissue factor decryption and microvesicle shedding. Blood Adv 2017;1(13): 849-862

31 Morrissey JH, Neuenschwander PF, Huang Q McCallum CD, Su B, Johnson AE. Factor VIIa-tissue factor: functional importance of protein-membrane interactions. Thromb Haemost 1997;78(01): 112-116

32 Morrissey JH, Tajkhorshid E, Sligar SG, Rienstra CM. Tissue factor/ factor VIIa complex: role of the membrane surface. Thromb Res 2012;129(Suppl 2):S8-S10

33 Ohkubo YZ, Morrissey JH, Tajkhorshid E. Dynamical view of membrane binding and complex formation of human factor VIIa and tissue factor. J Thromb Haemost 2010;8(05):1044-1053

$34 \mathrm{Ke} \mathrm{K}$, Yuan J, Morrissey JH. Tissue factor residues that putatively interact with membrane phospholipids. PLoS One 2014;9(02): e88675

35 Mallik S, Prasad R, Bhattacharya A, Sen P. Synthesis of phosphatidylserine and its stereoisomers: their role in activation of blood coagulation. ACS Med Chem Lett 2018;9(05):434-439

36 Zwaal RFA, Schroit AJ. Pathophysiologic implications of membrane phospholipid asymmetry in blood cells. Blood 1997;89 (04):1121-1132

37 Daleke DL. Regulation of transbilayer plasma membrane phospholipid asymmetry. J Lipid Res 2003;44(02):233-242

38 Fadeel B, Xue D. The ins and outs of phospholipid asymmetry in the plasma membrane: roles in health and disease. Crit Rev Biochem Mol Biol 2009;44(05):264-277

39 Zwaal RF, Comfurius P, Bevers EM. Surface exposure of phosphatidylserine in pathological cells. Cell Mol Life Sci 2005;62(09): 971-988

40 Kodigepalli KM, Bowers K, Sharp A, Nanjundan M. Roles and regulation of phospholipid scramblases. FEBS Lett 2015;589 (01):3-14

41 van der Mark VA, Elferink RP, Paulusma CC. P4 ATPases: flippases in health and disease. Int J Mol Sci 2013;14(04):7897-7922

42 Ruf W, Rehemtulla A, Morrissey JH, Edgington TS. Phospholipidindependent and -dependent interactions required for tissue factor receptor and cofactor function. J Biol Chem 1991;266 (04):2158-2166

43 Le DT, Rapaport SI, Rao LVM. Studies of the mechanism for enhanced cell surface factor VIIa/tissue factor activation of factor $\mathrm{X}$ on fibroblast monolayers after their exposure to N-ethylmaleimide. Thromb Haemost 1994;72(06):848-855

44 Ansari SA, Pendurthi UR, Sen P, Rao LV. The role of putative phosphatidylserine-interactive residues of tissue factor on its coagulant activity at the cell surface. PLoS One 2016;11(06): e0158377

45 Balasubramanian K, Mirnikjoo B, Schroit AJ. Regulated externalization of phosphatidylserine at the cell surface: implications for apoptosis. J Biol Chem 2007;282(25):18357-18364

46 Leonarduzzi G, Chiarpotto E, Biasi F, Poli G. 4-Hydroxynonenal and cholesterol oxidation products in atherosclerosis. Mol Nutr Food Res 2005;49(11):1044-1049
47 Mali VR, Palaniyandi SS. Regulation and therapeutic strategies of 4-hydroxy-2-nonenal metabolism in heart disease. Free Radic Res 2014;48(03):251-263

48 Vatsyayan R, Kothari H, Pendurthi UR, Rao LV. 4-Hydroxy-2nonenal enhances tissue factor activity in human monocytic cells via p38 mitogen-activated protein kinase activation-dependent phosphatidylserine exposure. Arterioscler Thromb Vasc Biol 2013;33(07):1601-1611

49 Ansari SA, Pendurthi UR, Rao LVM. The lipid peroxidation product 4-hydroxy-2-nonenal induces tissue factor decryption via ROS generation and the thioredoxin system. Blood Adv 2017;1(25): 2399-2413

50 Ebert J, Wilgenbus P, Teiber JF, et al. Paraoxonase-2 regulates coagulation activation through endothelial tissue factor. Blood 2018;131(19):2161-2172

51 Hagmann H, Kuczkowski A, Ruehl M, et al. Breaking the chain at the membrane: paraoxonase 2 counteracts lipid peroxidation at the plasma membrane. FASEB J 2014;28(04):1769-1779

52 Liang HPH, Hogg PJ. Critical importance of the cell system when studying tissue factor de-encryption. Blood 2008;112(03): 912-913

53 Langer F, Spath B, Fischer C, et al. Rapid activation of monocyte tissue factor by antithymocyte globulin is dependent on complement and protein disulfide isomerase. Blood 2013;121(12): 2324-2335

54 Furlan-Freguia C, Marchese P, Gruber A, Ruggeri ZM, Ruf W. P2 $\times 7$ receptor signaling contributes to tissue factor-dependent thrombosis in mice. J Clin Invest 2011;121(07):2932-2944

55 Wolberg AS, Monroe DM, Roberts HR, Hoffman MR. Tissue factor de-encryption: ionophore treatment induces changes in tissue factor activity by phosphatidylserine-dependent and -independent mechanisms. Blood Coagul Fibrinolysis 1999;10(04):201-210

56 Chen VM, Ahamed J, Versteeg HH, Berndt MC, Ruf W, Hogg PJ. Evidence for activation of tissue factor by an allosteric disulfide bond. Biochemistry 2006;45(39):12020-12028

57 Lysov Z, Swystun LL, Kuruvilla S, Arnold A, Liaw PC. Lung cancer chemotherapy agents increase procoagulant activity via protein disulfide isomerase-dependent tissue factor decryption. Blood Coagul Fibrinolysis 2015;26(01):36-45

58 Quinn PJ. Plasma membrane phospholipid asymmetry. Subcell Biochem 2002;36:39-60

59 Truman JP, Al Gadban MM, Smith KJ, Hammad SM. Acid sphingomyelinase in macrophage biology. Cell Mol Life Sci 2011;68(20): 3293-3305

60 Milhas D, Clarke CJ, Hannun YA. Sphingomyelin metabolism at the plasma membrane: implications for bioactive sphingolipids. FEBS Lett 2010;584(09):1887-1894

61 Pavoine C, Pecker F. Sphingomyelinases: their regulation and roles in cardiovascular pathophysiology. Cardiovasc Res 2009;82(02): 175-183

62 Wong ML, Xie B, Beatini N, et al. Acute systemic inflammation upregulates secretory sphingomyelinase in vivo: a possible link between inflammatory cytokines and atherogenesis. Proc Natl Acad Sci U S A 2000;97(15):8681-8686

63 Smith EL, Schuchman EH. The unexpected role of acid sphingomyelinase in cell death and the pathophysiology of common diseases. FASEB J 2008;22(10):3419-3431

64 Bianco F, Perrotta C, Novellino L, et al. Acid sphingomyelinase activity triggers microparticle release from glial cells. EMBO J 2009;28(08):1043-1054

65 Cremesti AE, Goni FM, Kolesnick R. Role of sphingomyelinase and ceramide in modulating rafts: do biophysical properties determine biologic outcome? FEBS Lett 2002;531(01):47-53

66 Hancock JF. Lipid rafts: contentious only from simplistic standpoints. Nat Rev Mol Cell Biol 2006;7(06):456-462

67 Mulder AB, Smit JW, Bom VJJ, et al. Association of smooth muscle cell tissue factor with caveolae. Blood 1996;88(04):1306-1313 
68 Mulder AB, Smit JW, Bom VJJ, Blom NR, Halie MR, van der Meer J. Association of endothelial tissue factor and thrombomodulin with caveolae. Blood 1996;88(09):3667-3670

69 Mandal SK, Pendurthi UR, Rao LVM. Cellular localization and trafficking of tissue factor. Blood 2006;107(12):4746-4753

70 Awasthi V, Mandal SK, Papanna V, Rao LV, Pendurthi UR. Modulation of tissue factor-factor VIla signaling by lipid rafts and caveolae. Arterioscler Thromb Vasc Biol 2007;27(06):1447-1455

71 Sevinsky JR, Rao LVM, Ruf W. Ligand-induced protease receptor translocation into caveolae: a mechanism for regulating cell surface proteolysis of the tissue factor-dependent coagulation pathway. J Cell Biol 1996;133(02):293-304

72 Dietzen DJ, Page KL, Tetzloff TA. Lipid rafts are necessary for tonic inhibition of cellular tissue factor procoagulant activity. Blood 2004;103(08):3038-3044

73 Goñi FM, Alonso A. Membrane fusion induced by phospholipase $C$ and sphingomyelinases. Biosci Rep 2000;20(06):443-463

74 López-Montero I, Monroy F, Vélez M, Devaux PF. Ceramide: from lateral segregation to mechanical stress. Biochim Biophys Acta 2010;1798(07):1348-1356

75 Sevier CS, Kaiser CA. Formation and transfer of disulphide bonds in living cells. Nat Rev Mol Cell Biol 2002;3(11):836-847

76 Mamathambika BS, Bardwell JC. Disulfide-linked protein folding pathways. Annu Rev Cell Dev Biol 2008;24:211-235

77 Liang HP, Brophy TM, Hogg PJ. Redox properties of the tissue factor Cys186-Cys209 disulfide bond. Biochem J 2011;437(03): 455-460

78 Schmidt B, Ho L, Hogg PJ. Allosteric disulfide bonds. Biochemistry 2006;45(24):7429-7433

79 Ahamed J, Versteeg HH, Kerver M, et al. Disulfide isomerization switches tissue factor from coagulation to cell signaling. Proc Natl Acad Sci U S A 2006;103(38):13932-13937

80 Reinhardt C, von Brühl ML, Manukyan D, et al. Protein disulfide isomerase acts as an injury response signal that enhances fibrin generation via tissue factor activation. J Clin Invest 2008;118(03): $1110-1122$

81 Ruf W, Versteeg HH. Tissue factor mutated at the allosteric Cys186-Cys209 disulfide bond is severely impaired in decrypted procoagulant activity. Blood 2010;116(03):500-501

82 van den Hengel LG, Kocatürk B, Reitsma PH, Ruf W, Versteeg HH. Complete abolishment of coagulant activity in monomeric disulfide-deficient tissue factor. Blood 2011;118(12):3446-3448

83 Kothari H, Nayak RC, Rao LV, Pendurthi UR. Cystine 186-cystine 209 disulfide bond is not essential for the procoagulant activity of tissue factor or for its de-encryption. Blood 2010;115(21):4273-4283
84 van den Hengel LG, Osanto S, Reitsma PH, Versteeg HH. Murine tissue factor coagulant activity is critically dependent on the presence of an intact allosteric disulfide. Haematologica 2013;98 (01):153-158

85 Zhou B, Hogg PJ, Gräter F. One-way allosteric communication between the two disulfide bonds in tissue factor. Biophys J 2017; 112(01):78-86

86 Prasad R, Banerjee S, Sen P. Contribution of allosteric disulfide in the structural regulation of membrane-bound tissue factor-factor VIla binary complex. J Biomol Struct Dyn 2018:1-14

87 Krudysz-Amblo J, Jennings ME II, Knight T, Matthews DE, Mann KG, Butenas S. Disulfide reduction abolishes tissue factor cofactor function. Biochim Biophys Acta 2013;1830(06):3489-3496

88 Wilkinson B, Gilbert HF. Protein disulfide isomerase. Biochim Biophys Acta 2004;1699(1-2):35-44

89 Jasuja R, Furie B, Furie BC. Endothelium-derived but not plateletderived protein disulfide isomerase is required for thrombus formation in vivo. Blood 2010;116(22):4665-4674

90 Cho J, Furie BC, Coughlin SR, Furie B. A critical role for extracellular protein disulfide isomerase during thrombus formation in mice. J Clin Invest 2008;118(03):1123-1131

91 Popescu NI, Lupu C, Lupu F. Extracellular protein disulfide isomerase regulates coagulation on endothelial cells through modulation of phosphatidylserine exposure. Blood 2010;116(06): 993-1001

92 Müller-Calleja N, Ritter S, Hollerbach A, Falter T, Lackner KJ, Ruf W. Complement C5 but not C3 is expendable for tissue factor activation by cofactor-independent antiphospholipid antibodies. Blood Adv 2018;2(09):979-986

93 Rothmeier AS, Marchese P, Langer F, et al. Tissue factor prothrombotic activity is regulated by integrin-arf6 trafficking. Arterioscler Thromb Vasc Biol 2017;37(07):1323-1331

94 Wang P, Wu Y, Li X, Ma X, Zhong L. Thioredoxin and thioredoxin reductase control tissue factor activity by thiol redox-dependent mechanism. J Biol Chem 2013;288(05):3346-3358

95 Rothmeier AS, Marchese P, Petrich BG, et al. Caspase-1-mediated pathway promotes generation of thromboinflammatory microparticles. J Clin Invest 2015;125(04):1471-1484

96 Lundström J, Holmgren A. Protein disulfide-isomerase is a substrate for thioredoxin reductase and has thioredoxin-like activity. J Biol Chem 1990;265(16):9114-9120

97 Bellisola G, Fracasso G, Ippoliti R, et al. Reductive activation of ricin and ricin A-chain immunotoxins by protein disulfide isomerase and thioredoxin reductase. Biochem Pharmacol 2004;67 (09):1721-1731 